\title{
Rotation Number as a Complete Topological Invariant of a Simple Isotopic Class of Rough Transformations of a Circle
}

\author{
E. V. Nozdrinova
}

The problem of the existence of a simple arc connecting two structurally stable systems on a closed manifold is included in the list of the fifty most important problems of dynamical systems. This problem was solved by S. Newhouse and M. Peixoto for Morse-Smale flows on an arbitrary closed manifold in 1980. As follows from the works of Sh. Matsumoto, P. Blanchard, V. Grines, E. Nozdrinova, and O. Pochinka, for the Morse-Smale cascades, obstructions to the existence of such an arc exist on closed manifolds of any dimension. In these works, necessary and sufficient conditions for belonging to the same simple isotopic class for gradient-like diffeomorphisms on a surface or a three-dimensional sphere were found. This article is the next step in this direction. Namely, the author has established that all orientation-reversing diffeomorphisms of a circle are in one component of a simple connection, whereas the simple isotopy class of an orientation-preserving transformation of a circle is completely determined by the Poincaré rotation number.

Keywords: rotation number, simple arc

Received November 05, 2018

Accepted November 27, 2018

The results for orientation-preserving maps are supported by RSF (Grant no. 17-11-01041), and the results for orientation-reversing maps are supported by the Basic Research Program at the National Research University Higher School of Economics (HSE) in 2018.

Elena V. Nozdrinova

maati@mail.ru

Laboratory of Topological Methods in Dynamics, NRU HSE

ul. Bolshaya Pecherskaya 25/12, Nizhny Novgorod, 603155 Russia

RUSSIAN JOURNAL OF NONLINEAR DYNAMICS, 2018, 14(4), 543-551 


\section{Introduction and formulation of results}

In the problems of managing processes of different nature of origin, every intermediate state of the controlled system is important. The control is effective if the transition of dynamics from one state to another is carried out in the simplest way. In particular, if the initial system changes its dynamics from one regular state to another, then it is natural to expect regularity from any intermediate state. The mathematical formulation of the problem of the existence of such a way is included in the list of the fifty most important problems of dynamical systems, compiled by J. Palis and C. Pugh [11].

The simplest regular systems are Morse-Smale systems - structurally stable flows and diffeomorphisms, whose nonwandering set consists of a finite number of orbits. Sh. Newhouse and M. Peixoto [8] proved that any Morse-Smale vector fields can be connected by a simple arc. Simplicity means that the arc consists of Morse-Smale systems only, except for a finite set of points (bifurcations) in which the vector field has (in some sense) the least deviations from the Morse-Smale system (see Section 2 for exact definitions).

For discrete dynamic systems, a fundamentally different situation is observed. We say that two isotopic diffeomorphisms belong to the same simple isotopic class if they are connected by a simple arc. From the works of Sh. Matsumoto [6] and P. Blanchard [2] it follows that any orientable closed surface admits Morse-Smale isotopic diffeomorphisms that cannot be connected by a simple arc. In [2] necessary and sufficient conditions (in terms of filtration) for belonging to the same simple isotopic class for gradient-like diffeomorphisms on an orientable surface are also established. V. Grines and O. Pochinka [3] found sufficient conditions for the existence of a simple arc connecting a gradient-like diffeomorphism on a 3-sphere with a "sourcesink" diffeomorphism. E. Nozdrinova and O. Pochinka [10] proved the existence of obstructions to the existence of a simple arc on nonsimply connected manifolds of any dimension.

In the present work, the following result is established.

Theorem 1. All rough orientation-reversing diffeomorphisms of a circle lie in one component of a simple connection, whereas the simple isotopy class of the rough orientation-preserving transformation of a circle is completely determined by the Poincaré rotation number.

\section{Supporting concepts and facts}

A. A. Andronov and L. S. Pontryagin [1] introduced the concept of rough systems of differential equations on the plane in 1937. By definition, such systems do not change the qualitative behavior with $C^{1}$ small changes to the right-hand sides of the equations. It turned out that rough systems have a very concise description: equilibrium states and limit cycles are hyperbolic, there is a finite number of them, and there are no trajectories going from saddle to saddle. The representative of the Gorky school, A. G. Mayer [5] introduced the concept of roughness for dynamic systems with discrete time (cascades) on a circle. From his results it follows that rough cascades on a circle, like flows on a plane, are typical and have fairly clear dynamics.

First of all, we recall that the circle $S^{1}$ is the only closed (compact without boundary) one-dimensional manifold and is homeomorphic to the standard unit circle

$$
\mathbb{S}^{1}=\left\{(x, y) \in \mathbb{R}^{2}: x^{2}+y^{2}=1\right\} .
$$

Rough diffeomorphisms $f$ of a circle $S^{1}$ (we denote the set of them by $G$ ) have dynamics which, from a modern point of view, can be described by the following proposition (see, for example, [4]). 
Proposition 1. Suppose that $f \in G$. Then

1) $f$ has a finite number (not less than two) of periodic points $p$, that is, points for which there is a natural number $m_{p}$ such that $f^{m_{p}}(p)=p$ and $f^{j}(p) \neq p$ for any integer $0<j<m_{p}$ (we denote by Per $(f)$ the set of periodic points of a diffeomorphism $f$ );

2 ) in the surrounding area $U_{p}$ of a point $p$, which is a connected component of the set $S^{1} \backslash(\operatorname{Per}(f) \backslash p)$, the diffeomorphism $f^{m_{p}}$ is topologically conjugated either to the expansion

$$
a_{ \pm}(x)= \pm 2 x: \mathbb{R}^{1} \rightarrow \mathbb{R}^{1}
$$

(in this case point $p$ is called a source, and the neighborhood $U_{p}$ is called its unstable manifold $W_{p}^{u}$ ) or to the contraction

$$
a_{ \pm}^{-1}(x)= \pm x / 2: \mathbb{R}^{1} \rightarrow \mathbb{R}^{1}
$$

(in this case point $p$ is called a sink, and the neighborhood $U_{p}$ is called its stable manifold $W_{p}^{s}$ ).

The immediate consequence of such a description of a rough transformation of the circle $f$ is the fact that a set $\operatorname{Per}(f)$ contains the same number (we denote this number by $r$ ) of sources and sinks that alternate on a circle, and the phase portrait $f$, up to periodicity, has the form shown in Fig. 1, where $\omega_{1}, \ldots, \omega_{r}$ are sinks, and $\alpha_{1}, \ldots, \alpha_{r}$ are sources. Connected components of a set $W_{\omega_{i}}^{s} \backslash \omega_{i}$ are called stable separatrices of the sink $\omega_{i}$, and connected components of a set $W_{\alpha_{i}}^{u} \backslash \alpha_{i}$ are called unstable separatrices of the source $\alpha_{i}$.

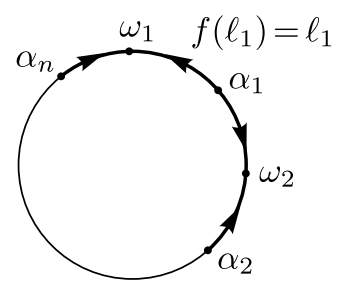

Fig. 1. Phase portrait of a rough circle transformation.

We assume that the circle is oriented clockwise and call the orientation on the $\operatorname{arc} \gamma \subset S^{1}$ positive (negative) if it coincides (does not coincide) with the orientation of the circle.

Let us recall that a homeomorphism $g: S^{1} \rightarrow S^{1}$ is called orientation-preserving (orientationreversing) if for any points $a, b, c \in S^{1}$ so that the point $b$ lies on a positively oriented arc $[a, c] \subset S^{1}$ it follows that the point $g(b)$ lies on a positive (negative) oriented arc $[g(a), g(c)]$ ).

We divide the set $G$ into two subclasses $G_{+}$and $G_{-}$, which consist of orientation-preserving and orientation-reversing diffeomorphisms, respectively. By [5] (see also [9]) periodic data of diffeomorphisms of class $G$ have the following structure.

\section{Proposition 2.}

1. For every diffeomorphism $f \in G_{+}$the set $\operatorname{Per}(f)$ consists of $2 n, n \in \mathbb{N}$ periodic orbits, each of which has period $k$.

2. For every diffeomorphism $f \in G_{-}$the set Per $(f)$ consists of $2 q, q \in \mathbb{N}$ periodic points, two of which are fixed, while others have period 2.

Renumber the periodic points of the set $\operatorname{Per}(f): p_{0}, p_{1}, \ldots, p_{2 n k-1}, p_{2 n k}=p_{0}$ are renumbered starting from an arbitrary periodic point $p_{0}$ clockwise. Then there is an integer $l$ so that $f\left(p_{0}\right)=p_{2 n l}$ and, what is more, $l=0$ for $k=1, l \in\{1, \ldots, k-1\}$ for $k>1$ and numbers $(k, l)$ are mutually simple. It is noticeable that $l$ is independent of the selection of point $p_{0}$. It is worth noting that the number $\frac{l}{k}$ coincides with the Poincaré rotation number in this case. 
It has to be remembered that the Poincaré rotation number for the orientation-preserving homeomorphism $f: S^{1} \rightarrow S^{1}$ is called the number $r(f) \in[0,1)$ defined as follows. Let us consider $\bar{f}: \mathbb{R} \rightarrow \mathbb{R}$ is the lift of homeomorphism $f$. Then for all $x \in \mathbb{R}$ there is a limit

$$
\rho(f)=\lim _{n \rightarrow \mathbb{N}} \frac{\bar{f}^{n}(x)-x}{n},
$$

which (up to a constant multiple $2 \pi$ ) does not depend on the choice of lift $\bar{f}$ and point $x$. Then the number

$$
r_{f}=\frac{\rho(f)}{2 \pi} \sim \bmod 1
$$

is called the Poincaré rotation number.

Note that the rotation number is an invariant of the homeomorphism $f$ with respect to orientation-preserving topological conjugacy. Let us call the triple $(n, k, l)$ periodic data of a diffeomorphism $f \in G_{+}$.

For $f \in G_{-}$we assume $\nu=-1 ; \nu=0 ; \nu=+1$ if its fixed points are sources; sink and source; sinks, respectively. It has to be observed that $\nu=0$ if $q$ is odd and $\nu= \pm 1$ if $q$ is even. Let us call the pair $(q, \nu)$ periodic data of a diffeomorphism $f \in G_{-}$.

According to the results of Mayer [5] (see also [9]), periodic data completely determine the topological conjugacy class (with respect to orientation-preserving conjugacy) of the diffeomorphism $f \in G$.

In this paper, we consider one-parameter families or arcs of diffeomorphisms $\phi_{t}, t \in[0,1]$, given on $S^{1}$. Denote by $\mathcal{Q}$ the set of $\operatorname{arcs}\left\{\phi_{t}\right\}$ with the end points at Morse-Smale diffeomorphisms and with the following properties:

1) $\phi_{t}$ has only finitely many orbits in their limit sets for every $t \in[0,1]$;

2) $\left\{\phi_{t}\right\}$ has only finitely many bifurcation values, say $b_{1}, \ldots, b_{m} \in(0,1)$.

In [7] it was proved that an $\operatorname{arc}\left\{\phi_{t}\right\} \in \mathcal{Q}$ is stable if it is simple, that is, for each $1 \leqslant i \leqslant s$, $\phi_{b_{i}}$ has no cycles and has exactly one non-hyperbolic periodic orbit, which is either a flip or a saddle-node (see Fig. 2).

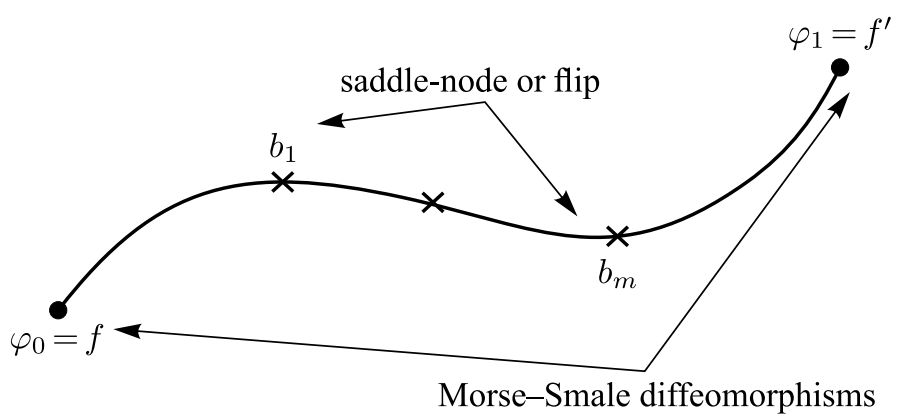

Fig. 2. A simple arc from the set $\mathcal{Q}$.

Thus, the assertion of Theorem 1 will follow from the following two lemmas, the proof of which is covered in the next section.

Lemma 1. Diffeomorphisms $f, f^{\prime} \in G_{+}$can be connected by a simple arc if they have the same rotation number.

Lemma 2. Any $f, f^{\prime} \in G_{-}$can always be connected by a simple arc. 


\section{Construction of a simple arc in the orientable case}

In this section we will prove Lemma 1.

Proof. Let $(n, k, l)$ be a triple of integers, so that $n \in \mathbb{N}$ and $l=0$ for $k=1, l \in\{1, \ldots, k-1\}$ for $k>1$, and numbers $(k, l)$ are coprime. We consider the map on the line $\bar{\Phi}$, given by the formula

$$
\bar{\Phi}_{n, k, l}(x)=x+\frac{1}{2 n k \pi} \sin (2 n k \pi x)+\frac{l}{k} .
$$

We assume $\overline{r_{i}}=\frac{i}{2 n k}, i \in \mathbb{Z}$. By construction, $\overline{r_{i}}$ are fixed points of the map $\bar{\Phi}-\frac{l}{k}$ (see Fig. 3). It is notable that arc $\bar{\phi}_{t}(x)=\bar{\Phi}_{n, k, l}\left(x+\frac{t}{2 n k}\right)-\frac{t}{2 n k}, t \in[0,1]$ connects a diffeomorphism $\bar{\Phi}_{n, k, l}$ with the diffeomorphism $\bar{\Phi}_{n, k, l}^{-1}$.

(a)

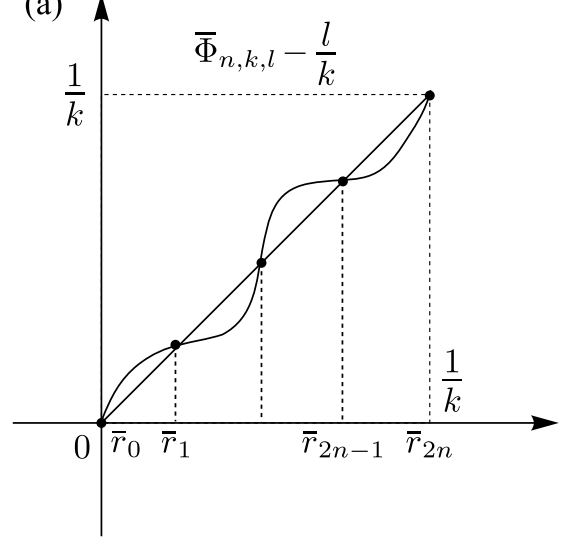

(b)

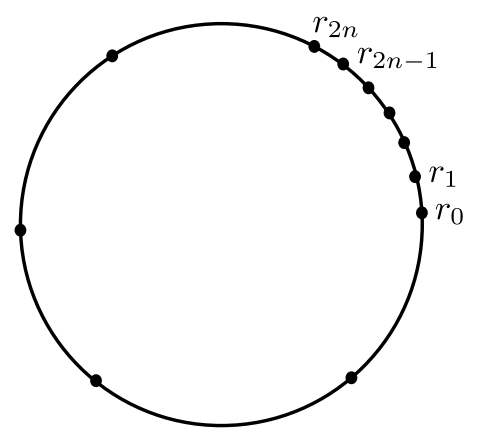

Fig. 3. (a) Map $\bar{\Phi}_{n, k, l}$; (b) map $\Phi_{n, k, l}$.

Let us consider projection $\Pi: \mathbb{R} \rightarrow \mathbb{S}^{1}$ given by the formula $\Pi(x)=e^{2 \pi i x}$. By the fact that the map $\bar{\Phi}$ is monotone increasing and periodic of period 1, it can be projected onto a circle by the diffeomorphism $\Phi_{n, k, l}=\Pi \bar{\Phi}_{n, k, l} \Pi^{-1}: \mathbb{S}^{1} \rightarrow \mathbb{S}^{1}$ (see Fig. 3). Let us call the diffeomorphism $\Phi_{n, k, l}$ a canonical diffeomorphism with periodic data $(n, k, l)$. Everywhere below, maps without a dash are projections onto $\mathbb{S}^{1}$ by means of $\Pi$ of maps with a dash on $\mathbb{R}$.

Now let $f$ be a diffeomorphism from the set $G_{+}$having periodic data $(n, k, l)$, and let $\bar{f}: \mathbb{R} \rightarrow \mathbb{R}$ be its lifting so that $\bar{f}(0) \in[0,1)$. Then the map $\bar{f}$ is monotone increasing and periodic of period 1 , in addition, the map $\bar{f}-\frac{l}{k}$ has $2 n$ fixed points $\bar{p}_{0}, \ldots, \bar{p}_{2 n-1}$ on the halfopen interval $[0,1)$. Without loss of generality we assume that $\bar{p}_{0}$ is a source (otherwise the diffeomorphism $f$ can be connected to a diffeomorphism with a simple arc $\Phi_{n, k, l}^{-1}$, and then connect the arc $H_{\Phi_{n, k, l}, \Phi_{n, k, l}^{-1}, t}^{-1}$ to the diffeomorphism $\left.\Phi_{n, k, l}\right)$.

We will construct a simple arc between the diffeomorphism $f$ and the canonical diffeomorphism $\Phi_{n, k, l}$. To do this, choose a strictly increasing smooth periodic function of period 1 $\bar{\varphi}: \mathbb{R} \rightarrow \mathbb{R}$, mapping points $\bar{r}_{i}$ to points $\bar{p}_{i}$ (see Fig. 4 ).

For all $t \in[0,1]$ it is assumed that

$$
\bar{\varphi}_{t}(x)=t \bar{\varphi}(x)+(1-t) x
$$




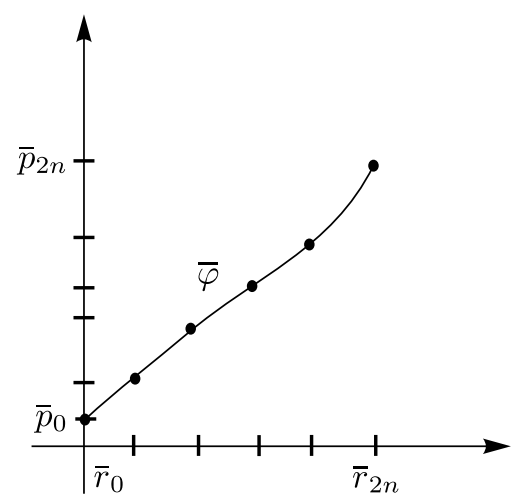

Fig. 4. Function $\bar{\varphi}$.

By construction, $\bar{\varphi}_{t}$ is a smooth isotopy connecting diffeomorphism $\bar{\varphi}$ with the identical map on the line. Then the arc

$$
\bar{\xi}_{t}=\bar{\varphi}_{t}^{-1}\left(\bar{f}-\frac{l}{k}\right) \bar{\varphi}_{t}
$$

connects the diffeomorphism $\bar{f}-\frac{l}{k}$ with the diffeomorphism $\bar{\xi}_{1}$ for which points $\bar{r}_{i}$ are fixed (see Fig. 5). Let us assume

$$
\bar{\eta}_{t}=t\left(\bar{\Phi}_{n, k, l}-\frac{l}{k}\right)+(1-t) \bar{\xi}_{1} .
$$

By construction, the arc

$$
\bar{H}_{\bar{f}, \bar{\Phi}_{n, k, l}, t}=\bar{\eta}_{t} * \bar{\xi}_{t}+\frac{l}{k}
$$

connects the diffeomorphism $\bar{f}$ with the canonical diffeomorphism $\bar{\Phi}_{n, k, l}$.

To construct an arc connecting diffeomorphisms $\bar{\Phi}_{n, k, l}$ and $\bar{\Phi}_{n-1, k, l}$, we consider a map $\bar{g}: \mathbb{R} \rightarrow \mathbb{R}$ (see Fig. 5) with period $\frac{1}{k}$ and given on the segment $\left[0, \frac{1}{k}\right]$ by the formula

$$
\bar{g}(x)= \begin{cases}x+\frac{3}{2 n k \pi} \sin \left(\frac{2 n k \pi x}{3}\right)+\frac{l}{k}, & 0 \leqslant x<\frac{3}{2 n k}, \\ \bar{\Phi}_{n, k, l}(x), & \frac{3}{2 n k} \leqslant x \leqslant \frac{1}{k} .\end{cases}
$$

Let (see Fig. 6)

$$
\bar{\zeta}_{t}=t \bar{g}+(1-t) \bar{\Phi}_{n, k, l} .
$$

By construction, the arc $H_{\Phi_{n, k, l}, \Phi_{n-1, k, l}, t}=H_{g, \Phi_{n-1, k, l}, t} * \zeta_{t}$ connects two canonical diffeomorphisms $\Phi_{n, k, l}, \Phi_{n-1, k, l}$ and contains a single saddle-node bifurcation point.

Now let us construct a simple arc between arbitrary diffeomorphisms $f, f^{\prime} \in G_{+}$with the same rotation numbers. In this case their periodic data $(n, k, l)$ and $\left(n^{\prime}, k^{\prime}, l^{\prime}\right)$ may differ only by the number of periodic orbits, i.e., $k=k^{\prime}$ and $l=l^{\prime}$. For definiteness we assume $n^{\prime} \leqslant n$. The arc connecting the canonical diffeomorphisms $\Phi_{n, k, l}$ and $\Phi_{n^{\prime}, k, l}$ is defined as follows:

$$
H_{\Phi_{n, k, l}, \Phi_{n^{\prime}, k, l}, t}=H_{\Phi_{n^{\prime}+1, k, l}, \Phi_{n^{\prime}, k, l}, t} * \ldots * H_{\Phi_{n, k, l}, \Phi_{n-1, k, l}, t} .
$$

Then we define the desired arc by the formula

$$
H_{f, f^{\prime}, t}=H_{f^{\prime}, \Phi_{n^{\prime}, k, l}, t}^{-1} * H_{\Phi_{n, k, l}, \Phi_{n^{\prime}, k, l}, t} * H_{f, \Phi_{n, k, l}, t} .
$$




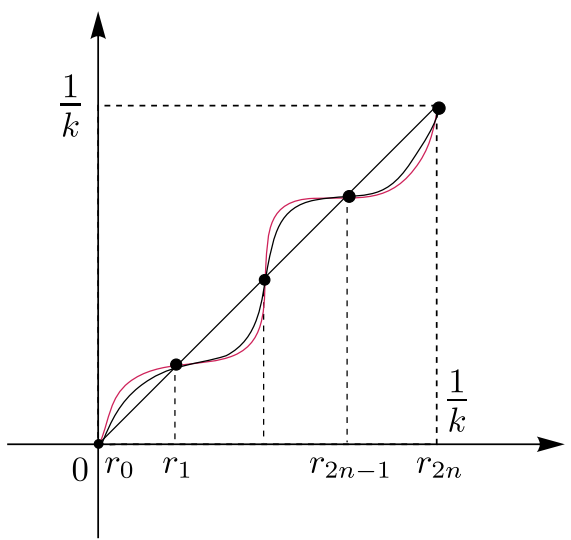

Fig. 5. Map $\bar{\Phi}_{n, k, l}-\frac{l}{k}$ and $\bar{\xi}_{1}$.

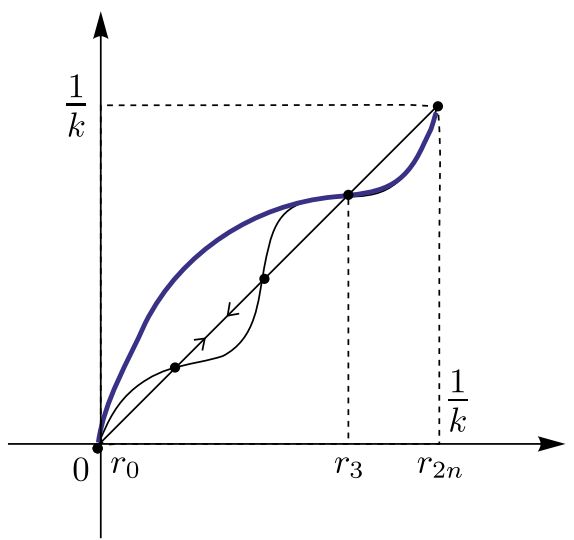

Fig. 6. Bifurcation saddle-node.

Thus, we have constructed a simple arc with a finite number of saddle-node bifurcations connecting diffeomorphisms $f, f^{\prime} \in G_{+}$with the same rotation numbers. Since the rotation number is a topological invariant of a circle diffeomorphism, continuously dependent on the parameter of the arc, it follows that any arc connecting diffeomorphisms $f, f^{\prime} \in G_{+}$with different rotation numbers is not simple, because it contains a continuum of bifurcations, which contradicts the definition of the simple arc.

\section{Construction of a simple arc in the nonorientable case}

In this section, Lemma 2 is to be proven.

Proof. Let us consider a map $\bar{\Psi}_{q}, q \in \mathbb{N}$ (see Fig. 7) given by the formula

$$
\bar{\Psi}_{q}(x)=-x-\frac{1}{2 q \pi} \sin (2 q \pi x) .
$$

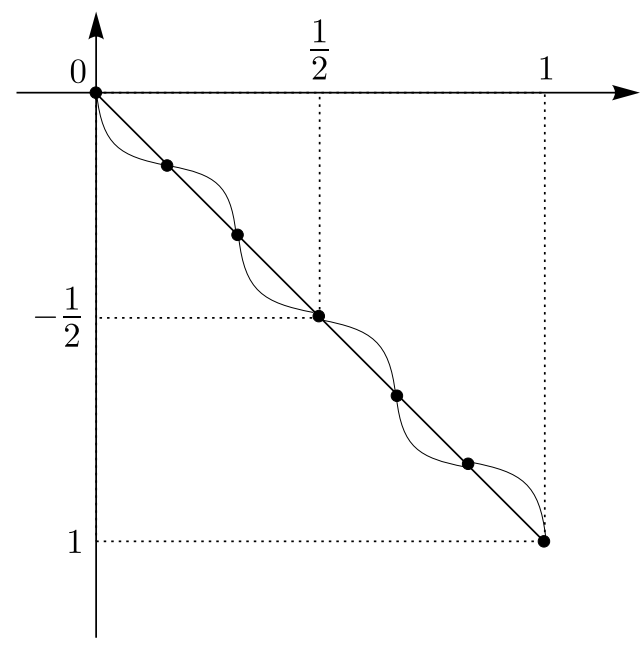

Fig. 7. Map $\bar{\Psi}_{3}$. 


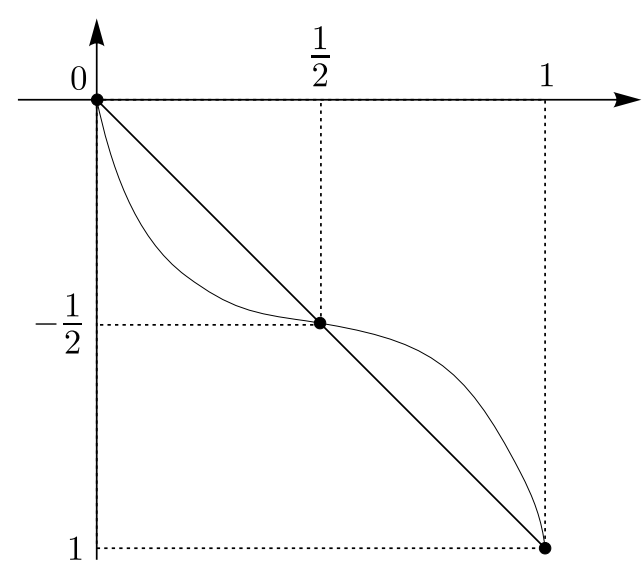

Fig. 8. Diffeomorphism $\bar{\Psi}_{1}$.

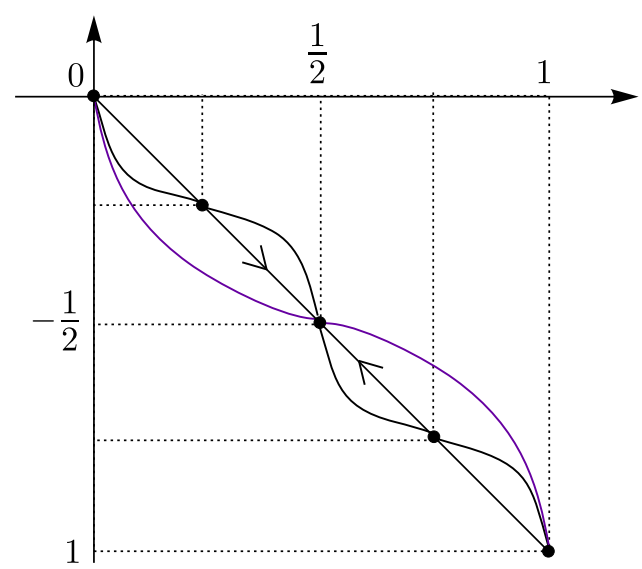

Fig. 9. Flipp bifurcation.

Notice that the map $\bar{\Psi}_{1}$ (see Fig. 8 ) is connected by an arc without bifurcations with the diffeomorphism $\bar{\Psi}_{1}^{-1}$ as follows:

$$
\bar{H}_{\bar{\Psi}_{1}, \bar{\Psi}_{1}^{-1}, t}(x)=\bar{\Psi}_{1}\left(x-\frac{t}{2}\right)+\frac{t}{2} .
$$

We also notice that the map $\bar{\Psi}_{2}$ is connected with the map $\bar{\Psi}_{1}$ by the simple $\operatorname{arc} \bar{H}_{\bar{\Psi}_{2}, \bar{\Psi}_{1}, t}=$ $=(1-t) \bar{\Psi}_{2}+t \bar{\Psi}_{1}$ with one flip bifurcation point (see Fig. 9).

As in the previous section, we will use notation without a dash for projections onto $\mathbb{S}^{1}$ by means of $\Pi$ of maps with a dash on $\mathbb{R}$. We call $\Psi_{q}$ a canonical diffeomorphism.

Let a diffeomorphism $f \in G_{-}$have periodical data $(q, \nu)$. We will show that the diffeomorphism $f$ is connected by a simple arc $H_{f, \Psi_{1}, t}$ with the diffeomorphism $\Psi_{1}$. Without loss of generality we will assume that the diffeomorphism $f$ has at least one fixed source (otherwise the diffeomorphism $f$ can be connected with the diffeomorphism $\Psi_{1}^{-1}$ by an algorithm below and then connected by the arc $H_{\Psi_{1}, \Psi_{1}^{-1}, t}^{-1}$ with the diffeomorphism $\Psi_{1}$ ).

A construction similar to that given in the previous section, an arc $H_{f, \Psi_{q}, t}$ without bifurcations, can be constructed to connect the diffeomorphism $f$ with the diffeomorphism $\Psi_{q}$. For $q \geqslant 3$, consider the map $\bar{h}: \mathbb{R} \rightarrow \mathbb{R}$ (see Fig. 10) with the period 1 defined on the segment $[0,1]$ by the formula

$$
\bar{h}(x)= \begin{cases}-x-\frac{3}{2 q \pi} \sin \left(\frac{2 q \pi x}{3}\right), & 0 \leqslant x<\frac{3}{2 q}, \\ \bar{\Psi}_{q}(x), & \frac{3}{2 q} \leqslant x \leqslant \frac{2 q-3}{2 q}, \\ -x+\frac{3}{2 q \pi} \sin \left(\frac{2 q \pi x}{3}\right), & \frac{2 q-3}{2 q}<x \leqslant 1 .\end{cases}
$$

Let us assume that $\bar{\varrho}_{t}=t \bar{h}+(1-t) \bar{\Psi}_{q}$. Then the arc

$$
H_{\Psi_{q}, \Psi_{q-2}, t}=H_{\varrho_{1}, \Psi_{q-2}, t} * \varrho_{t}
$$

joins two canonical diffeomorphisms $\bar{\Psi}_{q}$ and $\bar{\Psi}_{q-2}$ and contains one saddle-node bifurcation point. Thus, the required arc is given by

$$
H_{f, \Psi_{1}, t}=\left\{\begin{array}{l}
H_{\Psi_{3}, \Psi_{1}, t} * \ldots * H_{\Psi_{q}, \Psi_{q-2}, t} * H_{f, \Psi_{q}, t}, q=2 k+1, k \in \mathbb{N}, \\
H_{\Psi_{2}, \Psi_{1}, t} * H_{\Psi_{4}, \Psi_{2}, t} * \ldots * H_{\Psi_{q}, \Psi_{q-2}, t} * H_{f, \Psi_{q}, t}, q=2 k+2, k \in \mathbb{N} .
\end{array}\right.
$$




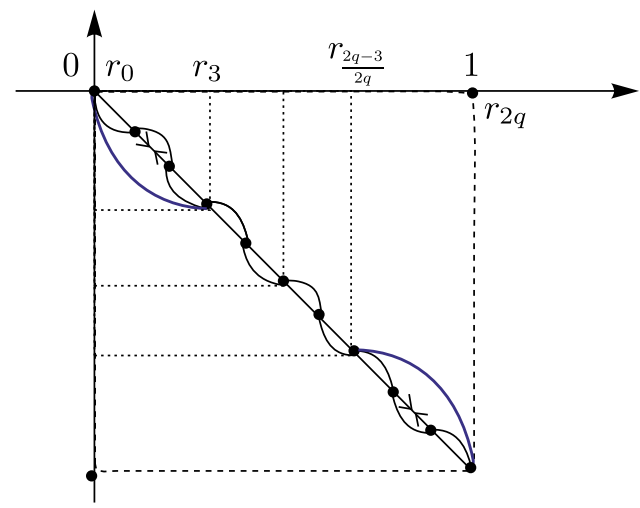

Fig. 10. Saddle-node bifurcation.

Acknowledgments. The author would like to thank Olga Pochinka for her attentive reading of the manuscript.

\section{References}

[1] Andronov, A. A. and Pontryagin, L. S., Rough Systems, Dokl. Akad. Nauk SSSR, 1937, vol. 14, no. 5, pp. 247-250 (Russian).

[2] Blanchard, P. R., Invariants of the NPT Isotopy Classes of Morse-Smale Diffeomorphisms of Surfaces, Duke Math. J., 1980, vol. 47, no. 1, pp. 33-46.

[3] Grines, V.Z. and Pochinka, O.V., On the Simple Isotopy Class of a Source-Sink Diffeomorphism on the 3-Sphere, Math. Notes, 2013, vol.94, nos. 5-6, pp.862-875; see also: Mat. Zametki, 2013, vol. 94 , no. 6, pp. 828-845.

[4] Grines, V., Medvedev, T., and Pochinka, O., Dynamical Systems on 2- and 3-Manifolds, Dev. Math., vol. 46, New York: Springer, 2016.

[5] Maier, A. G., Rough Transform Circle into a Circle, Uchen. Zap. Gorkov. Gos. Univ., 1939, no. 12, pp. 215-229 (Russian).

[6] Matsumoto, Sh., There Are Two Isotopic Morse-Smale Diffeomorphisms Which Cannot Be Joined by Simple Arcs, Invent. Math., 1979, vol.51, no. 1, pp.1-7.

[7] Newhouse, S., Palis, J., and Takens, F., Bifurcations and Stability of Families of Diffeomorphisms, Inst. Hautes Études Sci. Publ. Math., 1983, No. 57, pp. 5-71.

[8] Newhouse, S. and Peixoto, M. M., There Is a Simple Arc Joining Any Two Morse-Smale Flows, in Trois études en dynamique qualitative, Astérisque, vol. 31, Paris: Soc. Math. France, 1976, pp. 15-41.

[9] Pochinka, O. V., Nozdrinova, E. V., and Kolobianina, A. E., Classification of Rough Transformations of a Circle from a Modern Point of View, Zh. Srednevolzhsk. Mat. Obshch., 2017, vol.19, no.1, pp. 1-10 (Russian).

[10] Pochinka, O., Nozdrinova, E., and Dolgonosova, A., On the Obstructions to the Existence of a Simple Arc Joining the Multidimensional Morse-Smale Diffeomorphisms, Dinam. Sist., 2017, vol.7(35), no. 2, pp. 103-111.

[11] Palis, J. and Pugh, C. C., Fifty Problems in Dynamical Systems, in Dynamical Systems: Proc. Sympos. Appl. Topology and Dynamical Systems (Univ. Warwick, Coventry, 1973/1974): Presented to E. C. Zeeman on His Fiftieth Birthday, A. Manning (Ed.), Lecture Notes in Math., vol. 468, Berlin: Springer, 1975, pp. 345-353. 